Int. J. Odontostomat., 7(3):421-426, 2013.

\title{
Sinusitis Maxilar de Origen Odontogénica. Diagnóstico y Tratamiento Quirúrgico
}

\author{
Maxillary Sinusitis of Odontogenic Origin. Surgical Diagnosis and Treatment
}

\author{
Ferdinando De Conto; Mayara De Bona*; Graciele Rui*; \\ Gisele Rovani $^{* * *}$; Roque Rhoden ${ }^{* * * *}$ \& Mateus Ericson Flores ${ }^{* * * *}$
}

DE CONTO, F.; DE BONA, M.; RUI, G.; ROVANI, G.; RHODEN, R. \& FLORES, M. E. Sinusitis maxilar de origen odontogénica. Diagnóstico y tratamiento quirúrgico. Int. J. Odontostomat., 7(3):421-426, 2013.

RESUMEN: La sinusitis odontogénica afecta la mucosa de los senos maxilares y puede ser causada por la evolución de una lesión en el diente con formación de pus periapical que avanza en el interior de la cavidad sinusal. Se presenta un caso de un paciente con dens in dente en el diente incisivo lateral superior derecho, que sufrió el proceso de caries evolucionando en la región periapical, contaminando la mucosa sinusal de este lado, lo que causó el dolor, abultamiento y el desplazamiento del diente. La cirugía se realizó a través de acceso de Caldwell-Luc para el curetaje de la lesión, lo que indica la presencia en el diagnóstico histopatológico la presencia de quiste abscedado y sinusitis. Después de 18 meses de control, se observó la regeneración ósea y el reposicionamiento alveolar espontáneo de los dientes implicados. Los autores advierten que cíngulos profundos y dens in dente son las enfermedades de los dientes que merecen atención para el diagnóstico y la prevención de los procesos de caries con el fin de evitar complicaciones mayores.

PALABRAS CLAVE: dens in dente, seno maxilar, sinusitis odontogénica.

\section{INTRODUCCIÓN}

El seno maxilar es el más grande de los senos paranasales y es el primero que surge durante el desarrollo embrionario. Su revestimiento está constituido por un epitelio pseudo-estratificado columnar ciliado. Aunque las infecciones dentales son muy comunes, sólo el $5 \%$ a $10 \%$ causa sinusitis maxilar, lo que suele ocurrir debido a la interrupción de la membrana del seno maxilar, causada por el desplazamiento iatrogénico de diente en el seno maxilar, lesión periapical, enfermedad periodontal, lesiones de caries extensa o de extrusión de material obturador endodóntico dentro del seno (Brook, 2006; Costa et al., 2007; Rodrigues et al., 2009).

En general, las raíces de los premolares y molares se encuentran inmediatamente por debajo del piso del seno maxilar, lo que favorece la relación entre una anatomía patológica en estos dientes y la sinusi- tis. El diagnóstico de esta patología requiere un buen examen físico. Por lo general, los pacientes refieren una historia de infección respiratoria de vías aéreas superiores acompañando de dolor dental; también puede presentar alteración en el sonido nasal, dolor facial o cefalea, secreción nasal y tos durante la noche debido al paso de exudado en la faringe. También hay una sensibilidad dolorosa infraorbitaria a la palpación sobre el seno afectado. La historia de procedimiento dental reciente puede ayudar en el diagnóstico diferencial (Brook, 2006; Costa et al.; Mehra \& Murad, 2004; Bomeli et al., 2009).

El diagnóstico de la sinusitis odondogénica incluye una anamnesis detallada, un examen físico completo y pruebas de imágenes específicas, como la incidencia radiográfica Waters, lateral de cráneo, tomografía computarizada y exámenes radiográficos

Doctor en CTBMF, Profesor de Cirugía de la Facultad de Odontologia, Universidade de Passo Fundo (FOUPF), Grupo de Servicio Bucomaxilofacial del Hospital São Vicente de Paulo, Passo Fundo, Brasil.

* Académica, Facultad de Odontologia, Universidade de Passo Fundo (FOUPF), Passo Fundo, Brasil.

*** Mestre en Patología Bucal, Profesora de Estomatología, Universidade de Passo Fundo (FOUPF), Passo Fundo, Brasil.

**** Especialista en CTBMF, Grupo de Servicio Bucomaxilofacial del Hospital São Vicente de Paulo Passo Fundo, Brasil.

**t+*t Doctor en Radiología Odontológica, Universidade de Passo Fundo (FOUPF), Passo Fundo, Brasil. 
dentales (periapical, oclusal y ortopantomografia) (Vale et al., 2010). Los avances recientes en imagenología odontológica indican el uso de las imágenes computadorizadas tipo Cone-Beam, por la calidad de imagen y reducción en las dosis de radiación para el paciente (Nair \& Nair, 2010).

Los principales microorganismos que se encuentran en esta patología son las bacterias aeróbias y anaeróbias, con predominio de las anaeróbias Peptostreptococcus ssp., Fusobacterium spp., Prevotella spp. y Porphyromonas spp. En la mayoría de los casos, existe una correlación entre la flora existentes en la región periapical y la encontrada con una punción antral (Brook, 2005, 2006, 2009).

Uno de los cambios de desarrollo de los dientes es el dens in dente o dens invaginatus, el que afecta la superficie de desarrollo del elemento dental de manera que el diente se vuelva más vulnerable a las infecciones y caries (Alani \& Bishop, 2008). Debido a esta morfología, los dientes alterados pueden desarrollar caries de difícil percepción clínica, necrosis de la pulpa e infección periapical (Fregnani et al., 2008).

En el tratamiento de la sinusitis odontogénica es necesario actuar sobre la causa del diente y la enfermedad en sí misma. Sólo entonces, es posible eliminar la infección existente y prevenir las recurrencias o complicaciones. La combinación de la terapéutica farmacológica y quirúrgica, por lo general son necesarias para el tratamiento de la sinusitis odontogénica (Costa et al.; Vale et al.). El tratamiento no traumático preconizado para la sinusitis odontogénica es con los antibióticos de amplio espectro durante 3-4 semanas (Mehra \& Murad). La sinusitis sin tratar o mal tratada puede progresar a una variedad de complicaciones, tales como celulitis orbitaria, trombosis del seno cavernoso, meningitis, osteomielitis, absceso intracraneal y muerte (Brook, 2009).

\section{REPORTE DEL CASO}

Paciente sexo femenino de 14 años de edad, buscó atención odontológica en el servicio de ortodoncia, con queja de impactación del canino sobre el incisivo lateral maxilar derecho (Fig. 1). Después de una radiografía panorámica solicitada por el ortodontista, se observó imagen radiolúcida unilocular bien definida y amplia, con la participación de los ápices dentarios y la región del seno (Figs. 2 y 3). A con- tinuación, se derivó al Servicio de Cirugía Maxilofacial del Hospital São Vicente de Paulo, de Passo Fundo RS. La paciente refirió haber sufrido episodios de dolor local durante algún tiempo (un año), pero se encontraba asintomático en el momento. En su historia médica, refirió constantes tratamientos médicos para la sinusitis recurrente.

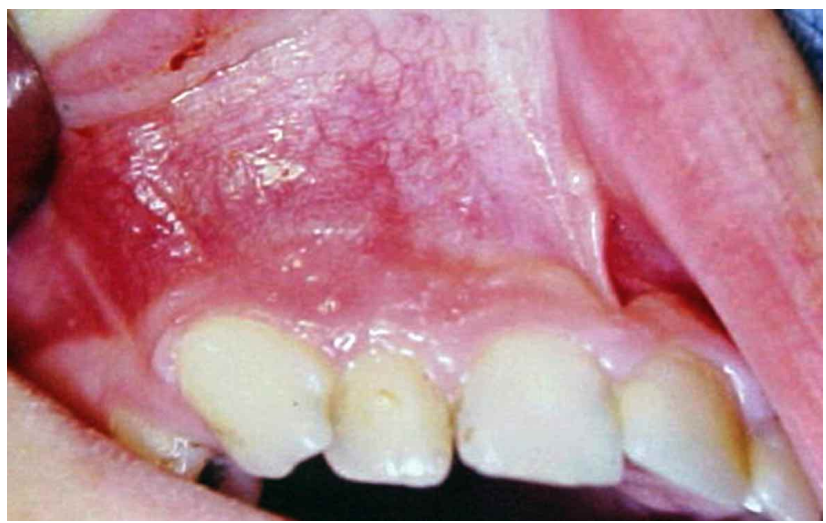

Fig. 1. Aspecto clínico que muestra el desplazamiento del canino superior derecho sobre el incisivo lateral.

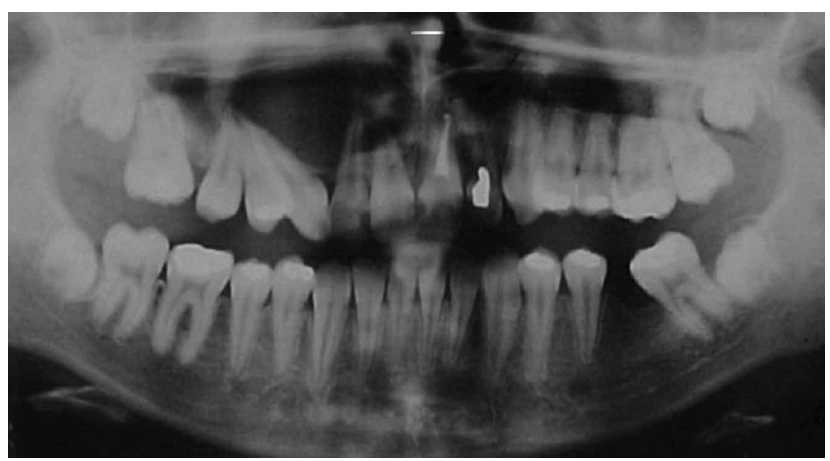

Fig. 2. Radiografía panorámica que muestra la extensión de la lesión radiotransparente y el desplazamiento del diente 13.

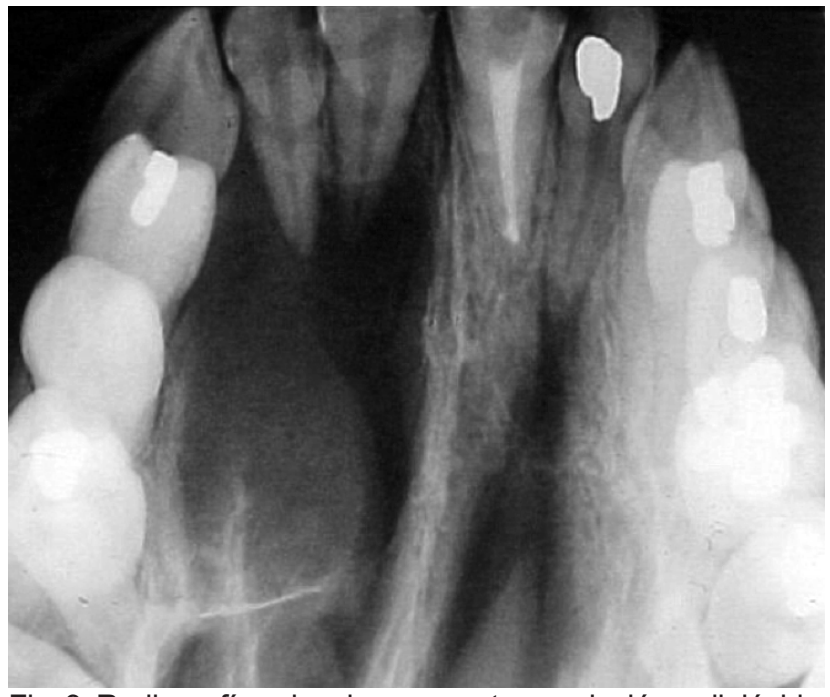

Fig. 3. Radiografía oclusal que muestra una lesión radiolúcida unilocular implicando la región apical de los dientes 11 al13. 
El examen clínico reveló el cambio de color en las coronas de los dientes 11 y 12 , y la prueba de la vitalidad fue negativo para ambos. En ambos dientes, se detectó la presencia de dens in dente del tipo I, con evidencia de lesiones de caries en los surcos. La mucosa regional no mostró ningún cambio. En el exámen de radiología panorámica se pudo observar tratamiento endodóntico en los dientes 21 y 22, y la presencia de una imagen radiotransparente con márgenes radioopacos en el periápice del diente 11 y otra imagen radiotransparente, unilocular bien definidos, que se extiende desde el ápice del diente 12 a la región del diente 16 , causando el desplazamiento de la cortical del suelo del seno maxilar y distorsión de la raíz del diente 13. La radiografia de Watters mostró opacificación del seno maxilar derecho.

Con base en el cuadro clínico, el hipótesis diagnóstica establecida fue de proceso infeccioso periapical de origen pulpar con posible comunicación con el seno maxilar derecho. La conducta para el caso entonces fue de solicitud de hemograma, lo cual acusó un desvío a la derecha para el número de leucocitos (leucocitosis), la paciente fue remitida para endodoncia de los dientes 11 y 12, y poco después la extirpación quirúrgica de la lesión.

Desde un acceso quirúrgico tipo Caldwell-Luc, se realizó la enucleación de la lesión, teniendo cuidado de no romper el tejido epitelial que la rodeaba y curetaje del ápice del diente 12. Además, se realizó la apicetomia en el diente 11. La paciente fue sometida a anestesia general debido a la extensión del procedimiento propuesto. En esta oportunidad se ha confirmado la comunicación entre la lesión de origen dental y el seno maxilar (Figs. 4 y 5). La lesión fue retirada y enviada al servicio de Patología de la Facultad de Odontología de la Universidade de Passo Fundo, RS, donde se describe una cavidad patológica con la presencia de supuración y material mucoso, a veces revestida por epitelio odontogénico, a veces por epitelio pseudoestratificado cilíndrico ciliado, además de infiltrado inflamatorio crónico superpuesto a cápsula fibrosa externa. El diagnóstico histopatológico fue de quiste radicular abscedado retirado del ápice del diente 12 y absceso crónico de la lesión retirada de la región periapical del diente 11.

El seguimiento clínico y radiográfico se llevó a cabo trimestralmente en el primer año, siendo observada regeneración ósea en el sitio después de 18 meses y, sobre todo, la curación del cuadro clínico radiográfico de la sinusitis, además de se observar el reposicionamiento del diente 13 sin la intervención de ortodoncia (Fig. 6).

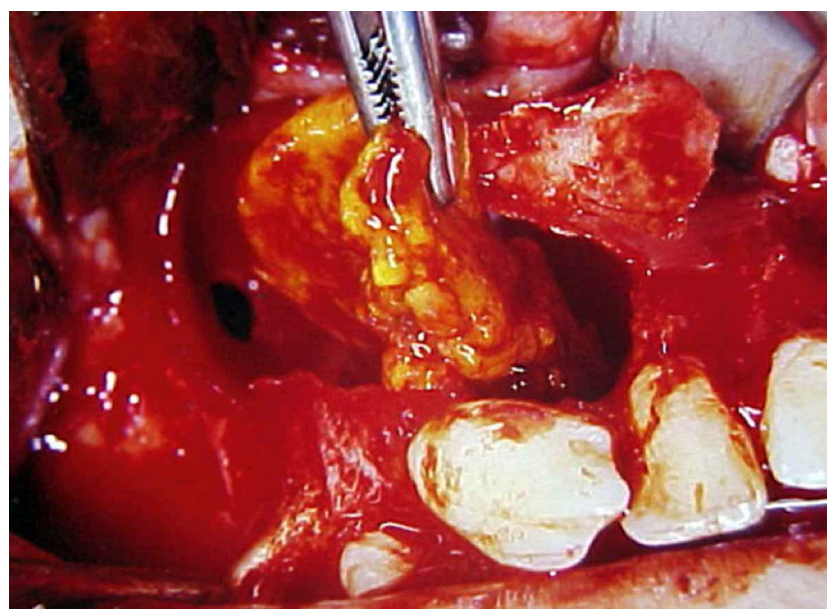

Fig. 4. El acceso quirúrgico y la enucleación de la lesión.

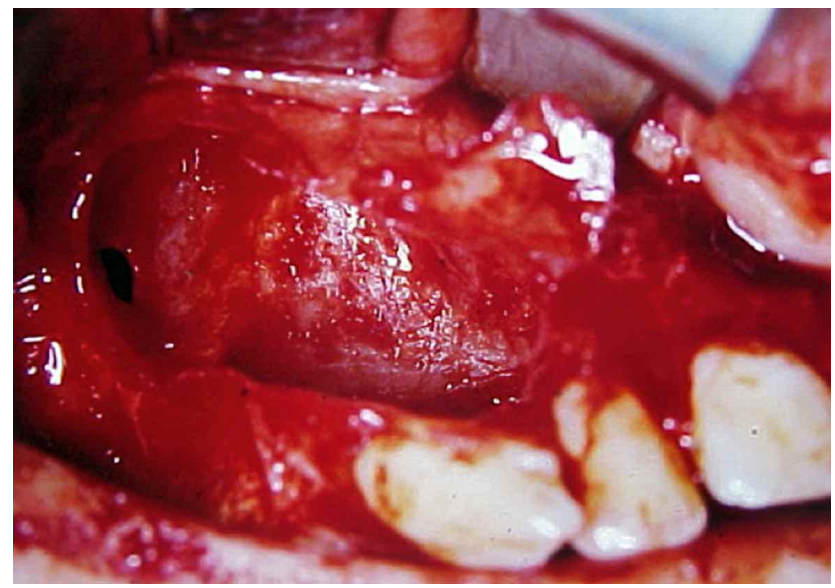

Fig. 5. Cavidad patológica. Tenga en cuenta la comunicación de la lesión con la porción anterior del seno maxilar.

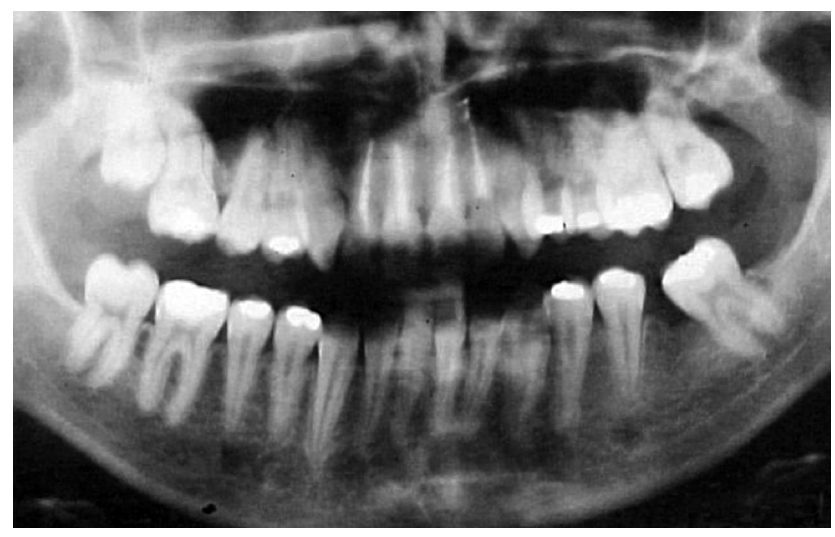

Fig. 6. Postoperatorio de 18 meses que muestran la regeneración ósea en el lugar, la reubicación del 13, la falta de opacidad en el seno maxilar y la endodoncia de los dientes 11 y 12. 


\section{DISCUSIÓN}

Las complicaciones de la sinusitis son importantes debido a la íntima relación de los senos paranasales con las cavidades craneanas y orbitarias, pues estas infección pueden causar complicaciones graves dentro de estas regiones. Los mecanismos patogénicos más comunes encontrados en estas complicaciones son las infecciones a través de la vía del hueso y la vascular. Entre las complicaciones características y comunes de los procesos sinusales se encuentran el mucocele, osteomielitis de los huesos del cráneo, meningitis, tromboflebitis del seno cavernoso, empiema epidural y subdural y el absceso cerebral (Brook, 2005, 2005). Las complicaciones orbitales corresponden al edema inflamatorio, celulitis, absceso orbitario, dacriocistitis purulenta (que es una supuración aguda del saco lagrimal y que puede ser secundaria a etmoiditis, sinusitis frontal o incluso maxilares) la y tromboflebitis de vena oftálmica (Brook, 2009).

El hallazgo radiológico más común y habitual es el engrosamiento de la mucosa sinusal maxilar tipo opacificación, con una incidencia del $10 \%$ de los casos (Vallo et al., 2010). Esta prueba se muestra para llevar a cabo la evidencia de alteraciones del suelo y de la pared posterior del seno maxilar. El diagnóstico de la sinusitis odontogénica, también puede ser ayudado por radiografías panorámicas convencionales o la TC de los senos faciales, muy utilizada debido a su alta capacidad y calidad en diagnosticar y evaluar el alcance de la sinusitis, así como su relación con otras estructuras anatómicas adyacentes (Vale et al.; Vallo et al.) Además, la TC no sólo ofrece la posibilidad de hacer un diagnóstico definitivo; también es fundamental para una mejor planificación terapéutica (Buyukkurt et al., 2010).

La TC por el método Cone-Beam es el medio de diagnóstico por imagen que más se ha desarrollado en los últimos años. Su aplicación en odontología es cada vez mayor para fines diagnósticos, planificación de tratamientos y su seguimiento (Nair \& Nair). En nuestro caso, las lesiones quísticas se pueden desarrollar lentamente, sin síntomas clínicos, los cuales aparecen al estar el seno maxilar involucrado. Por lo tanto, el diagnóstico se produce como hallazgo en el examen radiográfico dental, y en otros porque los síntomas corresponden convencionalmente a la sinusitis, con la transmisión de la presión a las paredes del seno maxilar, síntomas nasales e incluso se pue- de desarrollar algunos oftálmicos (Buyukkurt et al.). Células odontogénicas pueden ser precursoras de las lesiones quísticas que invaden el seno maxilar, como queratoquistes, ameloblastoma, odontoma, fibroma osificante, quiste dentígero y radicular, siendo este último lo que más comúnmente invade la cavidad del seno maxilar. La lesión periapical se encuentra principalmente a lo largo del ápice de los dientes que tienen lesiones de caries y la participación de la pulpa. Granulomas periapicales y abscesos pueden tener una imagen radiográfica similar (Mehra \& Murad).

Clasificar adecuadamente la sinusitis odontogénica según su etiología es una manera importante para definir el tipo de tratamiento que se debe utilizar. Las sinusitis odontogénicas se pueden clasificar como traumáticas (casos en que se vulnera de manera directa del suelo del seno maxilar como resultado de la extracción mal sucedida o accidentes traumáticos) y no traumática (causada por el avance de secreción de pus de origen dental al interior del seno maxilar) (Mehra et al., 1999). Siguiendo esta clasificación, el caso presentado se trata de una sinusitis odontogénica atraumática, pues se observó que la etiología del proceso infeccioso del seno maxilar (que podría causar más complicaciones), tuvo su origen en una lesión de caries en el cíngulo profundo de la cara palatina del incisivo lateral maxilar sin tratar, desde la cual se desarrolló una lesión pulpar que progresó hasta llegar a la cortical del suelo del seno maxilar derecho.

El dens invaginatus es una anomalía de desarrollo dentario, que afecta la dentaina y el esmalte. Técnicas de restauración o agentes sellantes están indicados para las áreas afectadas con invaginación del esmalte, con el fin de impedir la pérdida de vitalidad del diente. En los casos en que se pierde la vitalidad, el tratamiento endodóntico debe ser realizado (Aarsenaul et al., 2010). El tratamiento de la sinusitis de origen odontogénico usual requiere una combinación de tratamiento clínico y quirúrgico. Eliminar la causa (el resto de raíz dentro en el interior del seno o terapia endodóntica) es importante para prevenir la recurrencia de esta condición. El tratamiento clínico incluye 7-28 días de tratamiento antibiótico específico para la microflora del seno maxilar (Brook, 2006; Mehra \& Murad).

Aunque el tratamiento dental y drenaje quirúrgico son de suma importancia, la administración de la terapia antimicrobiana es esencial para ayudar a tratar 0 incluso prevenir las complicaciones (Brook, 2006). Basándose en estos informes, fue apropiado el abordaje 
quirúrgico para el caso descrito, ya que el paciente había recibido tratamiento médico sin obtener el resultado esperado, fue sometido a nuevo diagnóstico y tratamiento, eliminándose de este tiempo el foco inicial del periápice dentale y cureteando la lesión, inclusive del interior del seno maxilar. La técnica de Caldwell-Luc se define como una técnica para remover el tejido infectado del seno maxilar con acceso desde la pared lateral del seno maxilar sobre las raíces de los dientes restantes. Este procedimiento quirúrgico ofrece un buen postoperatorio y tiene pocas complicaciones. En los casos de sinusitis odontogénica, esta técnica es de elección debido a la facilidad de acceso al interior del seno maxilar (Huang \& Chen, 2011).

En estos casos, se debe señalar que la microflora de la sinusitis odontogénica es bastante variada y aun no está bien establecida, lo que hace que el tratamiento antimicrobiano sea generalmente iniciado empíricamente para atacar a los supuestos patógenos. También se observa en este caso, que la microbiota de este proceso infeccioso puede adquirir resistencia, y gradualmente hacer cambios en su constitución (Brook, 2006; Mehra \& Murad; Brook, 2009; Mehra et al.).

Un número creciente de bacterias gran-negativas anaeróbias (Fusobacterium spp. y Prevotella spp.) han adquirido resistencia a la producción de penicilina por la enzima beta-lactamasa. Los estudios demuestran que este tipo de microorganismo que puede afectar a cerca del $35 \%$ de los casos de sinusitis aguda y el $75 \%$ de tipo crónico. La penicilina se considera el fármaco de elección para el tratamiento de la sinusitis debido a su amplio espectro, sin embargo, el desarrollo de bacterias resistentes, limita su uso clínico. El paciente alérgico a este medicamento puede ser tratado con clindamicina o trimetoprimsulfametoxazol (Brook, 2006). Ante resistencia a la penicilina de los microorganismos en pacientes con sinusitis maxilar de origen odontogenico requiere la administración de agentes antimicrobianos eficaces contra estas bacterias. Esto incluye la clindamicina, cefalosporinas de segunda generación o carbapenem, e incluso una combinación de penicilina y un inhibidor de beta-lactamasa. El metronidazol se puede administrar si se asocia con un agente eficaz contra los estreptococos facultativos y aerobios (Brook, 2006).

\section{CONCLUSIONES}

Cíngulos profundos y dens in dente son alteraciones dentarias que merecen atención para el diagnóstico y la prevención de los procesos de caries con el fin de evitar más complicaciones. Los métodos radiográficos son importantes para confirmar el origen odontológico de la lesión sinusal. Sin embargo, los casos de sinusitis odontogénica atraumática pueden ser tratados con endodoncia y cirugía con curetaje como suplemento en los casos de lesiones extensas o persistencia de los signos y síntomas, siendo la técnica de Caldwell-Luc indicada para la intervención quirúrgicamente en estos casos.

DE CONTO, F.; DE BONA, M.; RUI, G.; ROVANI, G.; RHODEN, R. \& FLORES, M. E. Maxillary sinusitis of odontogenic origin. Surgical diagnosis and treatment. Int. J. Odontostomat., 7(3):421-426, 2013.

ABSTRACT: Odontogenic sinusitis affects mucous membrane of the maxillary sinus and can be caused by the evolution of a tooth injury with formation of periapical abscess that advances into the sinus cavity. We present a patient with dens in dente in the upper right lateral incisor tooth, which suffered carious process and periapical abscess, contaminating the sinus mucosa on this side, which caused pain, bulging and tooth displacement. Surgery was performed via a CaldwellLuc access for curettage of the lesion, indicating the presence in the histopathological diagnosis of radicular cyst abscess and sinusitis. After 18 months of control, alveolar bone regeneration and spontaneous repositioning of teeth involved was observed. The authors warn that cingulum deep in dens in dente are diseases of the tooth that deserve attention for the diagnosis and prevention of carious processes in order to avoid further complications.

KEY WORDS: dens in dente, maxillary sinus, sinusitis.

\section{REFERENCIAS BIBLIOGRÁFICAS}

Alani, A. \& Bishop, K. Dens invaginatus. Part 1: classification, prevalence and aetiology. Int. Endod. J., 41(12):112336, 2008.
Aarsenault, M.; Anderson, R. D.; Dyment, H.; Maclellan, J. \& Doyle, T. Facial cellulitis secondary to dens invaginatus: a case report. J. Can. Dent. Assoc., 76:a114, 2010. 
Bomeli, S. R.; Branstetter, B. F. 4th. \& Ferguson, B. J. Frequency of a dental source for acute maxillary sinusitis. Laryngoscope, 119(3):580-4, 2009.

Brook, I. Sinusitis of odontogenic origin. Otolaryngol. Head Neck Surg., 135(3):349-55, 2006.

Brook, I. Microbiology of Acute and Chronic Maxillary Sinusitis Associated with an Odontogenic Origin. Laryngoscope, 115(5):823-5, 2005.

Brook, I. Microbiology and antimicrobial treatment of orbital and intracranial complications of sinusitis in children and their management. Int. J. Pediatr. Otorhinolaryngol., 73(9):1183-6, 2009.

Buyukkurt, M. C.; Omezeli, M. M. \& Migoglu, O. Dentigerous cyst associate with an ectopic tooth in the maxillary sinus: A report of 3 cases and reniem of the literature. Oral Surg. Oral Med. Oral Pathol. Oral Radiol. Endod., 109(1):67-71, 2010.

Costa, F.; Emanuelli, E.; Robiony, M.; Zerman, N.; Polini, F. \& Politi, M. Surgical Treatment of Chronic Maxillary Sinusitis of Dental Origin. J. Oral Maxillofac. Surg., 65(2):223-8, 2007.

Fregnani, E. R.; Spinola, L. F.; Sônego, J. R.; Bueno, C. E. \& De Martin, A. S. Complex endodontic treatment of an immature type III dens invaginatus. A case report. Int. Endod. J., 41(10):913-9, 2008.

Huang, Y. C. \& Chen, W. H. Caldwell-Luc Operation Without Inferior Meatal Antrostomy: A Retrospective Study of 50 Cases. J. Oral Maxillofac. Surg., 70(9):2080-4, 2012.

Mehra, P. \& Murad, H. Maxillary sinus disease of odontogenic origin. Otolaryngol. Clin. North Am., 37(2):347-64, 2004.

Mehra, P.; Caiazzo, A. \& Bestgen, S. Odontogenic sinusitis causing orbital cellulitis. J. Am. Dent. Assoc., 130(7):1086-92, 1999.

Nair, U. P. \& Nair, M. K. Maxillary sinusitis of odontogenic origin: Cone-beam volumetric computerized tomography-aided diagnosis. Oral Surg. Oral Med. Oral Pathol. Oral Radiol. Endod., 110(6):e53-7, 2010.

Rodrigues, M. T. V.; Munhoz, E. A.; Cardoso, C. L.; Freitas, C. A. \& Damante, J. H. Chronic maxillary sinusitis associated with dental impression material. Med. Oral Pathol. Oral Cir. Bucal, 14(4):E163-6, 2009.

Vale, D. S.; Araujo, M. M.; Cavalieri, I.; Santos, M. B. P. \& Canellas, J.V.S. Sinusite maxilar de origem odontogênica: relato de caso. Rev. Port. Estomatol. Cir. Maxilofac., 51(2):141-6, 2010.
Vallo, J.; Suominen-Taipale, I.; Huumonen, S.; Soikkonen, K. \& Norblad, B. A. Prevalence of mucosal abnormalities of the maxillary sinus and their relationship to dental disease in panoramic radiography: results from the Health 2000 Health Examination survey. Oral Surg. Oral Med. Oral Pathol. Oral Radiol. Endod., 109(3):e80-7, 2010.

Dirección para Correspondencia:

Graciele Rui

Avenida Júlio de Castilho, 682 Sala 505

Bairro Centro

CEP: 95330-000

Veranópolis, RS

BRASIL

Teléfonos: 555434414233

Email: gra0503@hotmail.com

Recibido : 22-05-2013

Aceptado: 16-09-2013 